\title{
On the Generalized Hyers-Ulam-Rassias Stability of Higher Ring Derivations
}

\author{
KyOO-HONG PARK \\ Department of Mathematics Education, Seowon University, Chungbuk, Cheongju \\ 361-742, Korea \\ e-mail: parkkh@seowon.ac.kr \\ YONG-SoO JUNG* \\ Department of Mathematics, Sun Moon University, Asan, Chungnam 336-708, Ko- \\ rea \\ e-mail : ysjung@sunmoon.ac.kr
}

Abstract. We establish the generalized Hyers-Ulam-Rassias stability of higher ring derivations. Furthermore, we use the superstability of higher ring derivations to obtain approximately higher linear derivations mapping into the Jacobson radical under some conditions.

\section{Introduction and preliminaries}

The stability problem for a functional equation arising when we replace the functional equation by an inequality which acts as a perturbation of the equation, was raised by S.M. Ulam [12] in 1940, i.e., "Under what condition does there exists a homomorphism near an approximate homomorphism?" In the next year 1941, D.H. Hyers [7] was answered affirmatively the question of Ulam and the result can be formulated as follows: if $\varepsilon>0$ and $f: \mathcal{X} \rightarrow \mathcal{Y}$ is a map with $\mathcal{X}$ a normed space, $\mathcal{Y}$ a Banach space such that

$$
\|f(x+y)-f(x)-f(y)\| \leq \varepsilon
$$

for all $x, y \in \mathcal{X}$, then there exists a unique additive map $T: \mathcal{X} \rightarrow \mathcal{Y}$ such that

$$
\|f(x)-T(x)\| \leq \varepsilon
$$

for all $x \in \mathcal{X}$. Moreover, if $f(t x)$ is continuous in $t \in \mathbb{R}$ for each fixed $x$ in $\mathcal{X}$, where $\mathbb{R}$ denotes the real field, then $T$ is linear. This stability phenomenon is called the Hyers-Ulam stability of the additive functional equation $g(x+y)=g(x)+g(y)$.

* Corresponding author.

Received 20 February 2008; accepted 2 September 2008.

2000 Mathematics Subject Classification: 39B52, 46H99, 39B72, 39B82.

Key words and phrases: higher ring derivation, approximately higher ring derivation, stability. 
A generalized version of the theorem of Hyers for approximately additive maps was given by Th.M. Rassias [10] in 1978 by considering the case when the above inequality is not bounded: if there exist $a \theta \geq 0$ and $0 \leq p<1$ such that

$$
\|f(x+y)-f(x)-f(y)\| \leq \theta\left(\|x\|^{p}+\|y\|^{p}\right)
$$

for all $x, y \in \mathcal{X}$, then there exists a unique additive map $T: \mathcal{X} \rightarrow \mathcal{Y}$ such that

$$
\|f(x)-T(x)\| \leq \frac{2 \theta}{\left|2-2^{p}\right|}\|x\|^{p}
$$

for all $x \in \mathcal{X}$. From this fact, many authors say that the additive functional equation $g(x+y)=g(x)+g(y)$ has the Hyers-Ulam-Rassias stability property. In 1991, Z. Gajda [4] solved the problem for $p>1$, which was raised by Rassias. In fact, the result of Rassias is valid for $p>1$; moreover, Gajda gave an example that a similar stability result does not hold for $p=1$.

In 1992, a generalization of the Rassias theorem was obtained by P. Găvruţă [5]: Suppose $(\mathcal{G},+)$ is an abelian group, $\mathcal{Y}$ is a Banach space and the so-called admissible control function $\varphi: \mathcal{G} \times \mathcal{G} \rightarrow[0, \infty)$ satisfies

$$
\psi(x, y):=\frac{1}{2} \sum_{k=0}^{\infty} \frac{\varphi\left(2^{k} x, 2^{k} y\right)}{2^{k}}<\infty,
$$

for all $x, y \in \mathcal{G}$. If $f: \mathcal{G} \rightarrow \mathcal{Y}$ is a map such that

$$
\|f(x+y)-f(x)-f(y)\| \leq \varphi(x, y)
$$

for all $x, y \in \mathcal{G}$, then there exists a unique additive map $T: \mathcal{G} \rightarrow \mathcal{Y}$ such that

$$
\|f(x)-T(x)\| \leq \psi(x, x)
$$

for all $x \in \mathcal{G}$.

Throughout this paper, we assume that $\mathcal{A}$ and $\mathcal{B}$ are algebras over the real or complex field $\mathbb{F}$. An additive operator $h: \mathcal{A} \rightarrow \mathcal{B}$ is said to be a ring homomorphism if the functional equation $h(x y)=h(x) h(y)$ holds for all $x, y \in \mathcal{A}$. An additive operator $d: \mathcal{A} \rightarrow \mathcal{A}$ is said to be a ring derivation if the functional equation $d(x y)=x d(y)+d(x) y$ holds for all $x, y \in \mathcal{A}$. In addition, $d$ is called a linear derivation if the functional equation $d(\lambda x)=\lambda d(x)$ is valid for all $\lambda \in \mathbb{F}$ and all $x \in \mathcal{A}$.

Let $\mathbb{N}$ be the set of the natural numbers. From $m \in \mathbb{N} \cup\{0\}$, a sequence $H=\left\{h_{0}, h_{1}, \cdots, h_{m}\right\}$ (resp. $H=\left\{h_{0}, h_{1}, \cdots, h_{n}, \cdots\right\}$ ) of additive operators from $\mathcal{A}$ into $\mathcal{B}$ is called a higher ring derivation of rank $m$ (resp. infinite rank) from $\mathcal{A}$ into $\mathcal{B}$ if the functional equation

$$
h_{n}(z w)=\sum_{i=0}^{n} h_{i}(z) h_{n-i}(w) .
$$


holds for each $n=0,1, \cdots, m$ (resp. $n=0,1, \cdots$ ) and all $z, w \in \mathcal{A}$. If, in addition, each $h_{n}$ in $H$ satisfies the functional equation $h_{n}(\lambda x)=\lambda h_{n}(x)$ for all $\lambda \in \mathbb{F}$ and all $x \in \mathcal{A}$, then we will say that $H$ is just a higher linear derivation of rank $m$ (resp. infinite rank) from $\mathcal{A}$ into $\mathcal{B}$. A higher linear derivation $H$ from $\mathcal{A}$ into $\mathcal{B}$ is said to be continuous if each $h_{n}$ in $H$ is continuous on $\mathcal{A}$. A higher ring derivation $H$ of operators on $\mathcal{A}$, particularly, is called strong if $h_{0}$ is an identity operator. A higher ring derivation $H$ is said to be onto if $h_{0}$ maps $\mathcal{A}$ onto $\mathcal{B}$. Of course, a higher ring derivation of rank 0 from $\mathcal{A}$ into $\mathcal{B}$ (resp. a strongly higher derivation of rank 1 on $\mathcal{A}$ ) is a ring homomorphism (resp. a ring derivation). Note that a higher ring derivation is a generalization of both a ring homomorphism and a ring derivation. We say that a higher ring derivation $H$ on $\mathcal{A}$ which is strong maps $\mathcal{A}$ into its Jacobson radical if each $h_{n}$ in $H, n=1,2, \cdots$, maps $\mathcal{A}$ into its Jacobson radical.

Consider a sequence $F=\left\{f_{0}, f_{1}, \cdots, f_{m}\right\}$ (resp. $F=\left\{f_{0}, f_{1}, \cdots, f_{n}, \cdots\right\}$ ) of operators from $\mathcal{A}$ into $\mathcal{B}$ such that

$$
f_{n}(x+y+z w)=f_{n}(x)+f_{n}(y)+\sum_{i=0}^{n} f_{i}(z) f_{n-i}(w)
$$

for each $n=0,1, \cdots, m$ (resp. $n=0,1, \cdots$ ) and all $x, y, z, w \in \mathcal{A}$. For convenience' sake, we will say that the sequence $F$ is a higher derivation of rank $m$ (resp. infinite rank) from $\mathcal{A}$ into $\mathcal{B}$. In particular, if $f_{n}(0)=0$ for each $n=0,1, \cdots, m$ (resp. $n=0,1, \cdots$ ), then we see that $F$ is a higher ring derivation of rank $m$ (resp. infinite rank) from $\mathcal{A}$ into $\mathcal{B}$.

Example 1.1. Let $\mathcal{A}$ and $\mathcal{B}$ be algebras and $\alpha: \mathcal{A} \rightarrow \mathcal{B}$ a ring homomorphism. Let $a \in \mathcal{A}$ and $f: \mathcal{A} \rightarrow \mathcal{B}$ an operator defined by $f(z)=a \alpha(z)-\alpha(z) a$ for all $z \in \mathcal{A}$. Set $f_{0}=\alpha, f_{n}=0(1 \leq n \leq m-1), f_{m}=f$. Then it is clear that $f(z w)=\alpha(z) f(w)+f(z) \alpha(w)$ for all $z, w \in \mathcal{A}$. Now it is easy to see that $F=\left\{f_{0}, f_{1}, \cdots, f_{m}\right\}$ is a sequence of operators from $\mathcal{A}$ into $\mathcal{B}$ such that

$$
f_{n}(x+y+z w)=f_{n}(x)+f_{n}(y)+\sum_{i=0}^{n} f_{i}(z) f_{n-i}(w)
$$

for all $x, y, z, w \in \mathcal{A}$. That is, $F$ is a higher derivation of rank $m$ from $\mathcal{A}$ into $\mathcal{B}$ which in fact is a higher ring derivation since $f_{n}(0)=0$ for each $n=0,1, \cdots, m$.

In 1949, D.G. Bourgin [3] proved the following stability result, which is sometimes called the superstability of ring homomorphisms: Suppose that $\mathcal{A}$ and $\mathcal{B}$ are Banach algebras with unit. If $f: \mathcal{A} \rightarrow \mathcal{B}$ is a surjective map such that

$$
\begin{array}{r}
\|f(x+y)-f(x)-f(y)\| \leq \varepsilon \\
\|f(x y)-f(x) f(y)\| \leq \delta
\end{array}
$$

for some $\varepsilon>0, \delta>0$ and all $x, y \in \mathcal{A}$, then $f$ is a ring homomorphism. 
Recently, R. Badora [1] gave a generalization of the Bourgin's result. He [2] also obtained the following result for the Hyers-Ulam stability and the superstability of ring derivations: let $\mathcal{A}_{1}$ be a subalgebra of a Banach algebra $\mathcal{A}$. Assume that $f: \mathcal{A}_{1} \rightarrow \mathcal{A}$ is a map such that

$$
\begin{gathered}
\|f(x+y)-f(x)-f(y)\| \leq \varepsilon \\
\|f(x y)-x f(y)-f(x) y\| \leq \delta
\end{gathered}
$$

for some $\varepsilon \geq 0, \delta \geq 0$ and all $x, y \in \mathcal{A}_{1}$. Then there exists a unique ring derivation $d: \mathcal{A}_{1} \rightarrow \mathcal{A}$ such that

$$
\|f(x)-d(x)\| \leq \varepsilon
$$

for all $x \in \mathcal{A}_{1}$. Moreover,

$$
x\{f(y)-d(y)\}=0
$$

for all $x, y \in \mathcal{A}_{1}$. In addition, if $\mathcal{A}_{1}$ and $\mathcal{A}$ have the unit element, then $f$ is a ring derivation.

On the other hand, T. Miura et al. [9] proved the Hyers-Ulam-Rassias stability and the superstability of ring derivations on Banach algebras. Here it is natural to ask that there exists an approximately higher derivation which is not an exactly higher derivation. We observe the following example.

Example 1.2. Let $A$ be a compact Hausdorff space and let $C(A)$ be the commutative Banach algebra of complex-valued continuous functions on $A$ under pointwise operations and the supremum norm $\|\cdot\|_{\infty}$. Assume that $\vartheta: C(A) \rightarrow C(A)$ is a non-zero algebra homomorphism. We define $g: C(A) \rightarrow C(A)$ by

$$
g(z)(a)= \begin{cases}\vartheta(z)(a) \log |\vartheta(z)(a)| & \text { if } \vartheta(z)(a) \neq 0 \\ 0 & \text { if } \vartheta(z)(a)=0\end{cases}
$$

for all $z \in C(A)$ and $a \in A$. It is easy to see that $g(z w)=\vartheta(z) g(w)+g(z) \vartheta(w)$ for all $z, w \in C(A)$. Let $f_{0}=\vartheta, f_{n}=0,1 \leq n \leq m-1$ and $f_{m}=g$. Then we see that the sequence $F=\left\{f_{0}, f_{1}, \cdots, f_{m}\right\}$ satisfies the relation

$$
f_{n}(z w)=\sum_{i=0}^{n} f_{i}(z) f_{n-i}(w)
$$

for each $n=0,1, \cdots, m$ and all $z, w \in C(A)$. We claim that

$$
\left\|f_{n}(x+y+z w)-f_{n}(x)-f_{n}(y)-f_{n}(z w)\right\|_{\infty} \leq 4\left(1+\|x\|_{\infty}+\|y\|_{\infty}+\|z w\|_{\infty}\right)
$$

for all $x, y, z, w \in C(A)$. Indeed, since a non-zero algebra homomorphism on $C(A)$ has norm one, it follows that

$$
\left|f_{n}(x)(a)\right|=|g(x)(a)|=|\vartheta(x)(a)||\log | \vartheta(x)(a)|| \leq(1+|\vartheta(x)(a)|)^{2} \leq\left(1+\|x\|_{\infty}\right)^{2}
$$


for all $a \in C(A)$ with $\vartheta(x)(a) \neq 0$ and hence $\left\|f_{n}(x)\right\|_{\infty} \leq\left(1+\|x\|_{\infty}\right)^{2}$ for all $a \in C(A)$. Therefore we deduce that

$$
\left\|f_{n}(x+y+z w)-f_{n}(x)-f_{n}(y)-f_{n}(z w)\right\|_{\infty} \leq 4\left(1+\|x\|_{\infty}+\|y\|_{\infty}+\|z w\|_{\infty}\right)
$$

for all $x, y, z, w \in C(A)$. This yields

$$
\begin{aligned}
& \left\|f_{n}(x+y+z w)-f_{n}(x)-f_{n}(y)-\sum_{i=0}^{n} f_{i}(z) f_{n-i}(w)\right\|_{\infty} \\
= & \left\|f_{n}(x+y+z w)-f_{n}(x)-f_{n}(y)-f_{n}(z w)\right\|_{\infty} \\
\leq & 4\left(1+\|x\|_{\infty}+\|y\|_{\infty}+\|z w\|_{\infty}\right)
\end{aligned}
$$

for each $n=0,1, \cdots, m$ and all $x, y, z, w \in C(A)$. Hence we may regard $F$ as an approximately higher derivation of rank $m$ on $C(A)$.

\section{Main results}

Our goal is to investigate approximately higher derivations in the sense of the generalized version of Hyers-Ulam-Rassias due to [5]. Moreover, we are going to show the superstability of higher ring derivations.

Theorem 2.1. Let $\mathcal{A}$ be an algebra and $\mathcal{B}$ a Banach algebra. For each $n=$ $0,1,2, \cdots$, let $\varphi_{n}: \mathcal{A} \times \mathcal{A} \times \mathcal{A} \times \mathcal{A} \rightarrow[0, \infty)$ be a function such that

$$
\psi_{n}(x, y, z, w):=\frac{1}{2} \sum_{k=0}^{\infty} \frac{\varphi_{n}\left(2^{k} x, 2^{k} y, 2^{k} z, w\right)}{2^{k}}<\infty
$$

for all $x, y, z, w \in \mathcal{A}$. Suppose that $F=\left\{f_{0}, f_{1}, \cdots, f_{n}, \cdots\right\}$ is a sequence of operators from $\mathcal{A}$ into $\mathcal{B}$ such that each $n=0,1, \cdots$,

$$
\left\|f_{n}(x+y+z w)-f_{n}(x)-f_{n}(y)-\sum_{i=0}^{n} f_{i}(z) f_{n-i}(w)\right\| \leq \varphi_{n}(x, y, z, w)
$$

for all $x, y, z, w \in \mathcal{A}$. Then there exists a unique higher ring derivation $H=$ $\left\{h_{0}, h_{1}, \cdots, h_{n}, \cdots\right\}$ of any rank from $\mathcal{A}$ into $\mathcal{B}$ such that for each $n=0,1, \cdots$ and all $x \in \mathcal{A}$,

$$
\left\|f_{n}(x)-h_{n}(x)\right\| \leq \psi_{n}(x, x, 0,0)+c_{n},
$$

where

$$
c_{n}=\left\|\sum_{i=0}^{n} f_{i}(0) f_{n-i}(0)\right\|
$$

for each $n=0,1, \cdots$. Moreover,

$$
\sum_{i=0}^{n} h_{i}(z)\left\{f_{n-i}(w)-h_{n-i}(w)\right\}=0
$$


for each $n=0,1, \cdots$ and all $z, w \in \mathcal{A}$.

Proof. Putting $z=w=0$ in (2.2), we have

$$
\left\|f_{n}(x+y)-f_{n}(x)-f_{n}(y)-\sum_{i=0}^{n} f_{i}(0) f_{n-i}(0)\right\| \leq \varphi_{n}(x, y, 0,0)
$$

which yields

$$
\begin{aligned}
& \left\|f_{n}(x+y)-f_{n}(x)-f_{n}(y)\right\| \\
\leq & \left\|f_{n}(x+y)-f_{n}(x)-f_{n}(y)-\sum_{i=0}^{n} f_{i}(0) f_{n-i}(0)\right\|+\left\|\sum_{i=0}^{n} f_{i}(0) f_{n-i}(0)\right\| \\
\leq & \varphi_{n}(x, y, 0,0)+c_{n}
\end{aligned}
$$

for each $n=0,1, \cdots$ and all $x, y \in \mathcal{A}$. Using Hyers' direct method on inequality $(2.5)$, it follows from induction on $l$ that

$$
\left\|\frac{1}{2^{l}} f_{n}\left(2^{l} x\right)-f_{n}(x)\right\| \leq \frac{1}{2} \sum_{k=0}^{l-1} \frac{\varphi_{n}\left(2^{k} x, 2^{k} x, 0,0\right)+c_{n}}{2^{k}}
$$

for each $n=0,1, \cdots$ and all $x \in \mathcal{A}$ and that

$$
\left\|\frac{1}{2^{l}} f_{n}\left(2^{l} x\right)-\frac{1}{2^{m}} f_{n}\left(2^{m} x\right)\right\| \leq \frac{1}{2} \sum_{k=m}^{l-1} \frac{\varphi_{n}\left(2^{k} x, 2^{k} x, 0,0\right)+c_{n}}{2^{k}}
$$

for each $l>m$ and all $x \in \mathcal{A}$. Hence the convergence of (2.1) tells us that the sequence $\left\{\frac{1}{2^{l}} f_{n}\left(2^{l} x\right)\right\}$ is Cauchy for each $n=0,1, \cdots$ and all $x \in \mathcal{A}$. Let

$$
h_{n}(x):=\lim _{l \rightarrow \infty} \frac{1}{2^{l}} f_{n}\left(2^{l} x\right)
$$

for each $n=0,1, \cdots$ and all $x \in \mathcal{A}$. Taking $l \rightarrow \infty$ in (2.6), we obtain (2.3). In view of the same process as Hyers' method [7], we see that each operator $h_{n}, n=0,1, \cdots$, is additive and unique.

Next, we need to show that the sequence $H=\left\{h_{0}, h_{1}, \cdots, h_{n}, \cdots\right\}$ satisfies the identity

$$
h_{n}(z w)=\sum_{i=0}^{n} h_{i}(z) h_{n-i}(w)
$$

for each $n=0,1, \cdots$ and all $z, w \in \mathcal{A}$. Setting $x=y=0$ in $(2.2)$, we get

$$
\left\|f_{n}(z w)-2 f_{n}(0)-\sum_{i=0}^{n} f_{i}(z) f_{n-i}(w)\right\| \leq \varphi_{n}(0,0, z, w)
$$


which implies that

$$
\left\|f_{n}(z w)-\sum_{i=0}^{n} f_{i}(z) f_{n-i}(w)\right\| \leq \varphi_{n}(0,0, z, w)+2\left\|f_{n}(0)\right\|
$$

for each $n=0,1, \cdots$ and all $z, w \in \mathcal{A}$. Let a function $\Delta_{n}: \mathcal{A} \times \mathcal{A} \rightarrow \mathcal{B}$ be defined by

$$
\Delta_{n}(z, w)=f_{n}(z w)-\sum_{i=0}^{n} f_{i}(z) f_{n-i}(w)
$$

for each $n=0,1, \cdots$ and all $z, w \in \mathcal{A}$. Using (2.1) and (2.8), we have

$$
\lim _{l \rightarrow \infty} \frac{1}{2^{l}} \Delta_{n}\left(2^{l} z, w\right)=0
$$

for each $n=0,1, \cdots$ and all $z, w \in \mathcal{A}$. Now, from (2.7), (2.9) and (2.10), we deduce that

$$
\begin{aligned}
h_{n}(z w) & \left.=\lim _{l \rightarrow \infty} \frac{1}{2^{l}} f_{n}\left(2^{l}(z w)\right)=\lim _{l \rightarrow \infty} \frac{1}{2^{l}} f_{n}\left(\left(2^{l} z\right) w\right)\right) \\
& =\lim _{l \rightarrow \infty} \frac{1}{2^{l}}\left\{\sum_{i=0}^{n} f_{i}\left(2^{l} z\right) f_{n-i}(w)+\Delta_{n}\left(2^{l} z, w\right)\right\} \\
& =\lim _{l \rightarrow \infty} \sum_{i=0}^{n} \frac{1}{2^{l}} f_{i}\left(2^{k} z\right) f_{n-i}(w)+\lim _{l \rightarrow \infty} \frac{1}{2^{l}} \Delta_{n}\left(2^{k} z, w\right) \\
& =\sum_{i=0}^{n}\left\{\lim _{l \rightarrow \infty} \frac{1}{2^{l}} f_{i}\left(2^{l} z\right) f_{n-i}(w)\right\}=\sum_{i=0}^{n} h_{i}(z) f_{n-i}(w) .
\end{aligned}
$$

That is, we obtain that

$$
h_{n}(z w)=\sum_{i=0}^{n} h_{i}(z) f_{n-i}(w)
$$

for each $n=0,1, \cdots$ and all $z, w \in \mathcal{A}$. Let $l \in \mathbb{N}$ be fixed. Then, applying the relation (2.11) and the additivity of each $h_{n}, n=0,1, \cdots$, we get

$$
\begin{aligned}
\sum_{i=0}^{n} h_{i}(z) f_{n-i}\left(2^{l} w\right) & =h_{n}\left(z\left(2^{l} w\right)\right)=h_{n}\left(\left(2^{l} z\right) w\right) \\
& =\sum_{i=0}^{n} h_{i}\left(2^{l} z\right) f_{n-i}(w)=2^{l} \sum_{i=0}^{n} h_{i}(z) f_{n-i}(w) .
\end{aligned}
$$

Hence we get

$$
\sum_{i=0}^{n} h_{i}(z) f_{n-i}(w)=\sum_{i=0}^{n} h_{i}(z) \frac{1}{2^{l}} f_{n-i}\left(2^{l} w\right)
$$


for each $n=0,1, \cdots$ and all $z, w \in \mathcal{A}$. Taking $l \rightarrow \infty$ in (2.12), we see that

$$
\sum_{i=0}^{n} h_{i}(z) f_{n-i}(w)=\sum_{i=0}^{n} h_{i}(z) h_{n-i}(w)
$$

for each $n=0,1, \cdots$ and all $z, w \in \mathcal{A}$ which means (2.4). Combining (2.11) with (2.13), it follows that $H=\left\{h_{0}, h_{1}, \cdots, h_{n}, \cdots\right\}$ satisfies the relation

$$
h_{n}(z w)=\sum_{i=0}^{n} h_{i}(z) h_{n-i}(w) .
$$

for each $n=0,1, \cdots$ and all $z, w \in \mathcal{A}$. This completes the proof of the theorem.

Remark 2.2. Let $\mathcal{A}$ be an algebra and $\varphi_{n}: \mathcal{A} \times \mathcal{A} \times \mathcal{A} \times \mathcal{A} \rightarrow[0, \infty)$ a function such that

$$
\psi_{n}(x, y, z, w):=\frac{1}{2} \sum_{k=0}^{\infty} 2^{k} \varphi_{n}\left(2^{-k} x, 2^{-k} y, 2^{-k} z, w\right)<\infty
$$

for all $x, y, z, w \in \mathcal{A}$. If we replace (2.10) in Theorem by

$$
\lim _{l \rightarrow \infty} 2^{l} \Delta_{n}\left(2^{-l} z, w\right)=0,
$$

then (2.4) in Theorem does not generally hold in case of $\psi_{n}(x, y, z, w)<\infty$. For, we see that

$$
\lim _{l \rightarrow \infty} 2^{l} \Delta_{n}\left(2^{-l} z, w\right)=0
$$

is not valid since $\lim _{n \rightarrow \infty} 2^{l+1}\left\|f_{n}(0)\right\| \neq 0$.

Corollary 2.3. Let $\mathcal{A}$ be a normed algebra and $\mathcal{B}$ a Banach algebra. Let $\theta_{n} \in$ $(0, \infty)$ for each $n=0,1, \cdots$ and $p, q$ real numbers such that $p \neq 1$. Suppose that $F=\left\{f_{0}, f_{1}, \cdots, f_{n}, \cdots\right\}$ is a sequence of operators from $\mathcal{A}$ into $\mathcal{B}$ such that for each $n=0,1, \cdots$ and all $x, y, z, w \in \mathcal{A}$,

$\left\|f_{n}(x+y+z w)-f_{n}(x)-f_{n}(y)-\sum_{i=0}^{n} f_{i}(z) f_{n-i}(w)\right\| \leq \theta_{n}\left(\|x\|^{p}+\|y\|^{p}+\|z\|^{p}\|w\|^{q}\right)$.

Then there exists a unique higher ring derivation $H=\left\{h_{0}, h_{1}, \cdots, h_{n}, \cdots\right\}$ of any rank from $\mathcal{A}$ into $\mathcal{B}$ such that for each $n=0,1, \cdots$ and all $x \in \mathcal{A}$,

$$
\left\|f_{n}(x)-h_{n}(x)\right\| \leq \begin{cases}\frac{2 \theta_{n}}{2-2^{p}}\|x\|^{p}+c_{n} & \text { if } p<1, \\ \frac{2^{p} \theta_{n}}{2^{p}-2}\|x\|^{p}+c_{n} & \text { if } p>1\end{cases}
$$

where

$$
c_{n}=\left\|\sum_{i=0}^{n} f_{i}(0) f_{n-i}(0)\right\|
$$


for each $n=0,1, \cdots$. Moreover,

$$
\sum_{i=0}^{n} h_{i}(z)\left\{f_{n-i}(w)-h_{n-i}(w)\right\}=0
$$

for each $n=0,1, \cdots$ and all $z, w \in \mathcal{A}$.

Proof. $\varphi_{n}(x, y, z, w)=\theta_{n}\left(\|x\|^{p}+\|y\|^{p}+\|z\|^{p}\|w\|^{q}\right)$ for each $n=0,1, \cdots$ and all $x, y, z, w \in \mathcal{A}$. Suppose that $p<1$. Since

$$
\begin{aligned}
\psi_{n}(x, x, 0,0) & =\frac{1}{2} \sum_{k=0}^{\infty} \frac{\varphi_{n}\left(2^{k} x, 2^{k} x, 0,0\right)}{2^{k}} \\
& =\frac{\theta_{n}}{2} \sum_{k=0}^{\infty} \frac{\left\|2^{k} x\right\|^{p}+\left\|2^{k} x\right\|^{p}}{2^{k}} \\
& =\theta_{n}\|x\|^{p} \sum_{k=0}^{\infty} 2^{(p-1) k} \\
& =\theta_{n}\|x\|^{p} \frac{1}{1-2^{p-1}} \\
& =\frac{2 \theta_{n}}{2-2^{p}}\|x\|^{p},
\end{aligned}
$$

it follows from (2.3) in Theorem that

$$
\left\|f_{n}(x)-h_{n}\right\| \leq \psi_{n}(x, x, 0,0)+c_{n}=\frac{2 \theta_{n}}{2-2^{p}}\|x\|^{p}+c_{n} .
$$

for each $n=0,1, \cdots$ and all $x \in \mathcal{A}$. Next suppose that $p>1$. Since

$$
\begin{aligned}
\psi_{n}(x, x, 0,0) & =\frac{1}{2} \sum_{k=0}^{\infty} 2^{k} \varphi_{n}\left(2^{-k} x, 2^{-k} x, 0,0\right) \\
& =\frac{\theta_{n}}{2} \sum_{k=0}^{\infty} 2^{k}\left(\left\|2^{-k} x\right\|^{p}+\left\|2^{-k} x\right\|^{p}\right) \\
& =\theta_{n}\|x\|^{p} \sum_{k=0}^{\infty} 2^{(1-p) k} \\
& =\theta_{n}\|x\|^{p} \frac{1}{1-2^{1-p}} \\
& =\frac{2^{p} \theta_{n}}{2^{p}-2}\|x\|^{p},
\end{aligned}
$$

it follows from (2.3) in Theorem that

$$
\left\|f_{n}(x)-h_{n}\right\| \leq \psi_{n}(x, x, 0,0)+c_{n}=\frac{2^{p} \theta_{n}}{2^{p}-2}\|x\|^{p}+c_{n}
$$


for each $n=0,1, \cdots$ and all $x \in \mathcal{A}$.

By setting $\varphi_{n}(x, y, z, w)=\varepsilon_{n}$ for each $n=0,1, \cdots$ and all $x, y, z, w \in \mathcal{A}$, Theorem 2.1 also gives us the following corollary.

Corollary 2.4. Let $\mathcal{A}$ be an algebra and $\mathcal{B}$ a Banach algebra. Suppose that $F=$ $\left\{f_{0}, f_{1}, \cdots, f_{n}, \cdots\right\}$ is a sequence of maps from $\mathcal{A}$ into $\mathcal{B}$ such that for each $n=$ $0,1, \cdots$, there exists $\varepsilon_{n}>0$ such that

$$
\left\|f_{n}(x+y+z w)-f_{n}(x)-f_{n}(y)-\sum_{i=0}^{n} f_{i}(z) f_{n-i}(w)\right\| \leq \varepsilon_{n},
$$

for all $x, y, z, w \in \mathcal{A}$, then there exists a unique higher ring derivation $H=\left\{h_{0}, h_{1}\right.$, $\left.\cdots, h_{n}, \cdots\right\}$ of any rank from $\mathcal{A}$ into $\mathcal{B}$ such that for each $n=0,1, \cdots$ and all $x \in \mathcal{A}$,

$$
\left\|f_{n}(x)-h_{n}(x)\right\| \leq \varepsilon_{n}+c_{n},
$$

where

$$
c_{n}=\left\|\sum_{i=0}^{n} f_{i}(0) f_{n-i}(0)\right\|
$$

for each $n=0,1, \cdots$. Moreover, if $p<1$, then

$$
\sum_{i=0}^{n} h_{i}(z)\left\{f_{n-i}(w)-h_{n-i}(w)\right\}=0
$$

for each $n=0,1, \cdots$ and all $z, w \in \mathcal{A}$.

As a consequence of Corollary 2.4, we get the following Bourgin-type superstability [3] of higher ring derivations.

Theorem 2.5. Let $\mathcal{A}$ and $\mathcal{B}$ be Banach algebras with unit. Suppose that $F=$ $\left\{f_{0}, f_{1}, \cdots, f_{n}, \cdots\right\}$ is a sequence of operators from $\mathcal{A}$ into $\mathcal{B}$ satisfying $(2.14)$, where $f_{0}$ is onto. Then $F=\left\{f_{0}, f_{1}, \cdots, f_{n}, \cdots\right\}$ is a higher ring derivation of any rank from $\mathcal{A}$ into $\mathcal{B}$.

Proof. As in (2.5) and (2.8), the relation (2.14) yields that

$$
\left\|f_{n}(x+y)-f_{n}(x)-f_{n}(y)\right\| \leq \varepsilon_{n}+c_{n}
$$

for each $n=0,1, \cdots$ and all $x, y \in \mathcal{A}$, where

$$
c_{n}=\left\|\sum_{i=0}^{n} f_{i}(0) f_{n-i}(0)\right\|
$$

for each $n=0,1, \cdots$, and that

$$
\left\|f_{n}(z w)-\sum_{i=0}^{n} f_{i}(z) f_{n-i}(w)\right\| \leq \varepsilon_{n}+2\left\|f_{n}(0)\right\|
$$


for each $n=0,1, \cdots$ and all $z, w \in \mathcal{A}$. By induction, we lead the conclusion. From the Bourgin's theorem [3], we see that $f_{0}$ is a ring homomorphism from $\mathcal{A}$ onto $\mathcal{B}$ and so $(2.7)$ gives that $h_{0}(z)=\lim _{l \rightarrow \infty} \frac{1}{2^{l}} f_{0}\left(2^{l} z\right)=f_{0}(z)$ for all $z \in \mathcal{A}$, i.e., $f_{0}=h_{0}$. If $n=1$, then it follows from (2.15) that $f_{1}(z)=h_{1}(z)$ holds for all $z \in \mathcal{A}$ since $h_{0}$ is onto. Let us assume that $f_{m}(z)=h_{m}(z)$ is valid for all $z \in \mathcal{A}$ and all $m<n$. Then (2.15) implies that $h_{0}(z)\left\{f_{n}(w)-h_{n}(w)\right\}=0$ for all $z, w \in \mathcal{A}$. Since $h_{0}$ is onto, we have $f_{n}(w)=h_{n}(w)$ for all $w \in \mathcal{A}$. Hence we conclude that $f_{n}(z)=h_{n}(z)$ holds for all $n=0,1, \cdots$ and all $z \in \mathcal{A}$. Now Corollary2.4 tells us that $F=\left\{f_{0}, f_{1}, \cdots, f_{n}, \cdots\right\}$ is a higher ring derivation of any rank from $\mathcal{A}$ into $\mathcal{B}$. The proof of the theorem is complete.

We continue the next result.

Theorem 2.6. Let $\mathcal{A}$ be a Banach algebra with unit. Suppose that $F=\left\{f_{0}, f_{1}, \cdots\right.$, $\left.f_{n}, \cdots\right\}$ is a sequence of operators on $\mathcal{A}$ satisfying (2.14), where $f_{0}$ is an identity operator on $\mathcal{A}$. Then $F=\left\{f_{0}, f_{1}, \cdots, f_{n}, \cdots\right\}$ is a higher ring derivation of any rank on $\mathcal{A}$ which is strong.

Proof. By (2.7), we have

$$
h_{0}(x)=\lim _{l \rightarrow \infty} \frac{1}{2^{l}} f_{0}\left(2^{l} x\right)=x
$$

for all $x \in \mathcal{A}$ and so $h_{0}\left(=f_{0}\right)$ is an identity operator on $\mathcal{A}$. From the same method as in the proof of Corollary 2.4 using the induction and the relation (2.15), we get

$$
z\left\{f_{n}(w)-h_{n}(w)\right\}=0
$$

for all $n \in \mathbb{N}$ and all $z, w \in \mathcal{A}$. Since $\mathcal{A}$ contains the unit, it follows that $f_{n}(w)=$ $h_{n}(w)$ for all $n \in \mathbb{N}$ and all $w \in \mathcal{A}$. Thus, by Corollary 2.4, we see that $F=$ $\left\{f_{0}, f_{1}, \cdots, f_{n}, \cdots\right\}$ is a higher ring derivation of any rank on $\mathcal{A}$ which is strong. This completes the proof.

I.M. Singer and J. Wermer [11] obtained a fundamental result in a Banach algebra theory which started investigation into the ranges of linear derivations on Banach algebras. The result, which is called the Singer-Wermer theorem, states that every continuous linear derivation on a commutative Banach algebra maps into the Jacobson radical. K.-W. Jun and Y.-W. Lee [8] generalized the Singer-Wermer theorem to higher linear derivations which are strong and continuous. That is, every higher linear derivation of rank $m$ which is strong and continuous on a commutative Banach algebra maps into its Jacobson radical. The following is the Jun and Lee's result for approximately higher derivations in the sense of Hyers-Ulam.

Theorem 2.6. Let $\mathcal{A}$ be a commutative Banach algebra with unit. Suppose that $F=\left\{f_{0}, f_{1}, \cdots, f_{m}\right\}$ is a sequence of continuous operators on $\mathcal{A}$ such that for each $n=0,1, \cdots, m$, there exists $\varepsilon_{n}>0$ such that

$$
\left\|f_{n}(\alpha x+\beta y+z w)-\alpha f_{n}(x)-\beta f_{n}(y)-\sum_{i=0}^{n} f_{i}(z) f_{n-i}(w)\right\| \leq \varepsilon_{n}
$$


for all $x, y, z, w \in \mathcal{A}$ and all $\alpha, \beta \in \mathbb{U}=\{z \in \mathbb{C}:|z|=1\}$, where $f_{0}$ is an identity operator on $\mathcal{A}$. Then $F$ maps $\mathcal{A}$ into its Jacobson radical.

Proof. Put $\alpha=\beta=1 \in \mathbb{U}$ in (2.16). Then it follows from Corollary 2.4 that $F$ is a higher ring derivation of rank $m$ on $\mathcal{A}$ which is continuous. Setting $y=x$, $z=w=0$ in (2.16) and then following the same process as in (2.5), we obtain that

$$
\left\|f_{n}((\alpha+\beta) x)-(\alpha+\beta) f_{n}(x)\right\| \leq \varepsilon_{n}+c_{n}
$$

for each $n=0,1, \cdots, m$ and all $x \in \mathcal{A}$, where

$$
c_{n}=\left\|\sum_{i=0}^{n} f_{i}(0) f_{n-i}(0)\right\|
$$

for each $n=0,1, \cdots, m$. Thus we see that

$$
\frac{1}{2^{l}}\left\|f_{n}\left(2^{l}(\alpha+\beta) x\right)-(\alpha+\beta) f_{n}\left(2^{l} x\right)\right\| \rightarrow 0
$$

as $l \rightarrow \infty$ which implies that for each $n=0,1, \cdots, m$,

$$
h_{n}((\alpha+\beta) x)=\lim _{l \rightarrow \infty} \frac{1}{2^{l}} f_{n}\left(2^{l}(\alpha+\beta) x\right)=(\alpha+\beta) \lim _{l \rightarrow \infty} \frac{1}{2^{l}} f_{n}\left(2^{l} x\right)=(\alpha+\beta) h_{n}(x)
$$

for all $x \in \mathcal{A}$ and all $\alpha, \beta \in \mathbb{U}$.

Clearly, $h_{n}(0 x)=0=0 h_{n}(x)$ for each $n=0,1, \cdots, m$ and all $x \in \mathcal{A}$. Now, let $\lambda \in \mathbb{C}(\lambda \neq 0)$, and let $N \in \mathbb{N}$ greater than $|\lambda|$. By appying a geometric argument, we see that there exist $\lambda_{1}, \lambda_{2} \in \mathbb{U}$ such that $2 \frac{\lambda}{N}=\lambda_{1}+\lambda_{2}$. By the additivity of each $h_{n}, n=0,1, \cdots, m$, we get $h_{n}\left(\frac{1}{2} x\right)=\frac{1}{2} h_{n}(x)$ for each $n=0,1, \cdots, m$ and all $x \in \mathcal{A}$.

Therefore, we have

$$
\begin{aligned}
h_{n}(\lambda x) & =h_{n}\left(\frac{N}{2} \cdot 2 \cdot \frac{\lambda}{N} x\right)=N h_{n}\left(\frac{1}{2} \cdot 2 \cdot \frac{\lambda}{N} x\right)=\frac{N}{2} h_{n}\left(\left(\lambda_{1}+\lambda_{2}\right) x\right) \\
& =\frac{N}{2}\left(\lambda_{1}+\lambda_{2}\right) h_{n}(x)=\frac{N}{2} \cdot 2 \cdot \frac{\lambda}{N} h_{n}(x)=\lambda h_{n}(x)
\end{aligned}
$$

for each $n=0,1, \cdots, m$ and all $x \in \mathcal{A}$, so that $h_{n}$ is $\mathbb{C}$-linear for each $n=$ $0,1, \cdots, m$. This means that that $F=\left\{f_{0}, f_{1}, \cdots, f_{m}\right\}$ is a higher linear derivation of rank $m$ which is strong and continuous on $\mathcal{A}$. The Jun and Lee's result now guarantees that $F$ maps $\mathcal{A}$ into its Jacobson radical. This completes the proof.

Corollary 2.7. Let $\mathcal{A}$ be a commutative semisimple Banach algebra with unit. Suppose that $F=\left\{f_{0}, f_{1}, \cdots, f_{m}\right\}$ is a sequence of continuous operators on $\mathcal{A}$ such that for each $n=0,1, \cdots, m$, there exists $\varepsilon_{n}>0$ such that

$$
\left\|f_{n}(\alpha x+\beta y+z w)-\alpha f_{n}(x)-\beta f_{n}(y)-\sum_{i=0}^{n} f_{i}(z) f_{n-i}(w)\right\| \leq \varepsilon_{n}
$$


for all $x, y, z, w \in \mathcal{A}$ and all $\alpha, \beta \in \mathbb{U}=\{z \in \mathbb{C}:|z|=1\}$, where $f_{0}$ is an identity operator on $\mathcal{A}$. Then $f_{n}=0$ for each $n=1,2, \cdots, m$.

\section{References}

[1] R. Badora, On approximate ring homomorphisms, J. Math. Anal. Appl., 276(2)(2002), 589-597.

[2] R. Badora, On approximate derivations, Math. Inequal. Appl., 9(1)(2006), 167-173.

[3] D. G. Bourgin, Approximately isometric and multiplicative transformations on continuous function rings, Duke Math. J., 16(1949), 385-397.

[4] Z. Gajda, On stability of additive mappings, Internat. J. Math. Math. Sci., 14(1991), 431-434.

[5] P. Găvruță, A generalization of the Hyers-Ulam-Rassias stability of approximately additive mappings, J. Math. Anal. Appl., 184(1994), 431-436.

[6] O. Hatori and J. Wada, Ring derivations on semi-simple commutative Banach algebras, Tokyo J. Math., 15(1992), 223-229.

[7] D. H. Hyers, On the stability of the linear functional equation, Proc. Natl. Acad. Sci., 27(1941), 222-224.

[8] K.-W. Jun and Y.-W. Lee, The image of a continuous strong higher derivation is contained in the radical, Bull. Korean Math. Soc., 33(1996), 229-233.

[9] T. Miura, G. Hirasawa and S.-E. Takahasi, A perturbation of ring derivations on Banach algebras, J. Math. Anal. Appl., 319(2006), 522-530.

[10] Th. M. Rassias, On the stability of the linear mapping in Banach spaces, Proc. Amer. Math. Soc., 72(1978), 297-300.

[11] I. M. Singer and J. Wermer, Derivations on commutative normed algebras, Math. Ann., 129(1955), 260-264.

[12] S. M. Ulam, A Collection of Mathematical Problems, Interscience Publ., New York, 1960. 